\title{
Antibiotic susceptibility pattern and prevalence of Staphylococcus aureus from Patients specimens at Ayub Medical Complex Abbottabad, Pakistan
}

\author{
Sheryar Jamil, Muhammad Azam Khan", Zahid Ahmad, Sajjad Ali and \\ Iffraz Ali Syed \\ Department of Microbiology Government Post Graduate College Mandian Abbotabad-Pakistan \\ *Corresponding author's email:microbiologistazam@gmail.com

\section{Citation} \\ Sheryar Jamil, Muhammad Azam Khan, Zahid Ahmad, Sajjad Ali and Iffraz Ali Syed. Antibiotic susceptibility \\ pattern and prevalence of Staphylococcus aureus from Patients specimens at Ayub Medical Complex Abbottabad, \\ Pakistan. Pure and Applied Biology. Vol. 9, Issue 1, pp269-274. http://dx.doi.org/10.19045/bspab.2020.90031
}

\begin{tabular}{llll}
\hline \hline Received: 18/07/2019 & Revised: 19/09/2019 & Accepted: 25/09/2019 & Online First: 23/10/2019 \\
\hline
\end{tabular}

\section{Abstract}

Staphylococcus aureus causing wide spectrum of infections and has tendency for the development of multidrug resistance. The study was conducted to find out the antibiogram of $S$. aureus from clinical samples at Ayub Teaching institute, Abbottabad since September 2018 to May 2019 and to determine the prevalence of MRSA and MSSA.Total 100 Staphylococcus aureus was collected from clinical samples (pus, urine and blood) and identification was done by standard microbiological methods and antibiotic susceptibility testing was done by using disk diffusion method as per CLSI guidelines. Out of these 100 isolates, 44 (44\%) were MRSA and $56(56 \%)$ were MSSA. Most of the MRSA isolates were collected from blood 20(45.45\%) followed by pus $14(31.81 \%)$ and urine $10(22.7 \%)$ correspondingly while the highest prevalence of MSSA was in pus samples $26(46.42 \%)$, and were same in the samples of blood and urine $15(26.78 \%)$. MRSA was sensitive to Amikacin (95\%) and Vancomycin (86\%) but resistant to Oxacillin (100\%) and Imipenem (82\%). MSSA was sensitive to Oxacillin (100\%) and Doxycyclin (86\%) but resistant to Ciprofloxacin and Oflaxacin (64\%). All the isolates were sensitive to Amikacin, Doxycyclin and Gentamicin .Frequency of S.aureus is common in clinical samples of patients at Abbottabad and these were momentously resistant to frequently prescribed antibiotics, so, anti-staphylococcal antibiotics correct use is crucial.

Keywords: Methicillin resistant Staphylococcus aureus; Methicillin sensitive Staphylococcus aureus; Susceptibility; Staphylococcus aureus prevalence

\section{Introduction}

Staphylococcus aureus is a Gram-positive bacterium that reside upper respiratory tract, mouth, skin and conjunctiva and the major source of both nosocomial and opportunistic infections. It has the potential to develop resistance against antibiotics by several mechanisms including, inactivation by the enzymes, by variation in target site, and antibiotic trapping [1]. Due to these reasons it results in soft tissue infections, bone infection, skin infection, urinary tract 
infections, pneumonia, both in hospital settings and community [2]. This pathogen also form biofilm in wound which is challenging to treat [3]. It produces alpha, beta, gamma and delta hemolysis [4].

Methicillin Resistant strains are frequent cause of hospital acquired infections and are responsible for illness and death [5]. It was first identified in 1960 and was documented b-lactamase resistant and recognized by British scientists in 1961. The first MRSA in US was known in 1968 [6]. Due to misuse of antibiotics the predominance of MRSA has increased in medical care units which has resulted massive incidence of mortality and morbidity through the last two decades. Due to MRSA Approximately ten thousand and nine hundred deceases annually have been described in the US, more than those due to AIDS. Its toxicities can only be cured with certain categories of antibacterial agents. [7]. The incidence of MRSA differs among countries and has been reported all over the world. As compared to northern Europe its incidence in Pakistan and India is higher [8]. In 2007 A study reported 42-51\% prevalence of MRSA [9]. A comparable study from two hospitals in Rawalpindi during 2011 presented MRSA value of $53.3 \%$ [10]. Its prevalence and antibiotic resistance has created tasks for clinicians and the treatment of common infections have become problematic it takes lengthier time to control the infections triggered by risky bacteria [11]. Antibiogram is better process than molecular techniques as it is very simple, effortlessly accessible, having low cost, and carried out without any trouble. That is why when there is unavailability of molecular methods microbiologists and chemists practice it as a mention guide to clinic particular or public specific resistance pattern [12]. In Europe this method is still used for the epidemiological researches of MARSA [13]. Susceptibility of bacteria to medicines fluctuates from area to area, this research was carried out to evaluate the occurrence of MRSA and MSSA and S. aureus and of antibiotic susceptibility to the repeatedly recommended antibiotics in Ayub Teaching institute.

\section{Materials and methods Study area}

This study was conducted from September 2018 to May 2019 at Ayub Medical Complex, Abbottabad, Pakistan

\section{Samples Collection}

A total of 600 medical specimens including pus, urine and blood collected in sterilized bottles and swabs and processed in microbiology laboratory at Ayub Medical Complex Hospital, Abbottabad

\section{Samples culturing}

All the samples were cultured on CLED, MacConkey, and Blood agar (Oxoid, England) and incubated for $24 \mathrm{hrs}$ at $37^{\circ} \mathrm{C}$. 100 S.aureus isolates were recognized by performing Standard microbiological techniques such as, Gram staining and biochemical tests.

\section{Antimicrobial susceptibility}

To determine antibiotic susceptibility S.aureus were Sub cultured on Muller Hinton at $37^{\circ} \mathrm{C}$ for $24 \mathrm{hrs}$ and disc diffusion method was used as per CLSI standards.

\section{Antibiotics used in the study}

Levofloxacin $(30 \mu \mathrm{g})$, Ciprofloxacin $(30 \mu \mathrm{g})$, Of laxacin $(30 \mu \mathrm{g})$,Erythromycin $(30 \mu \mathrm{g})$,Doxycy clin( $30 \mu \mathrm{g})$, Gentamicin $(30 \mu \mathrm{g})$, Amikacin $(30$

$\mu \mathrm{g})$, Cephradine $(30 \mu \mathrm{g})$ Imipenem(30 $\mu \mathrm{g})$, Mer openem( $30 \mu \mathrm{g})$,Vancomycin( $30 \mu \mathrm{g})$, Amoxici

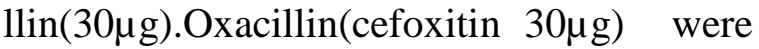
used for MRSA screening. The zone size greater or equal to $21 \mathrm{~mm}$ was dignified resistant and a zone size greater than $22 \mathrm{~mm}$ was dignified susceptible. Isolates were considered as susceptible, or resistant according to CLSI guidelines. As a control Staphylococcus aureus ATCC 25923 was taken. 


\section{Results}

A total of $100 \mathrm{~S}$. aureus isolates were explored of which $40(40 \%)$ were from pus, $35(35 \%)$ from blood and $25(25 \%)$ from urine. Among these 100 samples $(n=100)$ $44(44 \%)$ were resistant to oxacilin (MRSA) and $56(56 \%)$ were sensitive to oxacilin (MSSA). Out of 44 MRSA isolates 20(45\%), $14(31.81 \%)$ and $10(22.7 \%)$, were isolated from blood, pus and urine respectively. On the other hand out of the total $56(56 \%)$ isolates of MSSA the highest prevalence was detected in pus $26(46.42 \%)$ followed by urine $15(26.78 \%)$ and blood $15(26.78 \%)$ respectively (Table 1 ).

Antimicrobial susceptibility profile of staphylococcus aureus

Antibiogram of Staphylococcus aureus was studied. All isolates were from patients' clinical specimens (mainly pus, urine, blood) and were evaluated according to CLSI guidelines standards. $S$. aureus isolates were sensitive to Amikacin (86\%) followed by Doxycyclin (78\%), Vancomycin (76\%) and Gentamicin (72\%). While resistant to Oflaxacin, (76\%) followed by Ciprofloxacin (68\%) imipenum (62\%) and Erythromycin $(54 \%)$ respectively as presented in the (Table

2). The antimicrobial susceptibility pattern of antibiotics was further explained between both MARSA (resistant to oxacillin) and MSSA (sensitive to oxacillin).

\section{Antimicrobial susceptibility profile of MRSA and MSSA}

MRSA isolated from different samples were highly sensitive to Amikacin (95\%) followed by Vancomycin (86\%), Doxycycline (68\%) Gentamycin (59\%) and was resistance to Oxacillin (100\%) Imipenem (82\%) ciprofloxacin (73\%), Oflaxacin (71\%), Erythromycin (68\%), Amoxilin Levofloxacin (64\%) and meropenem (59\%) respectively.

MSSA exhibited significant sensitivity to Oxacillin (100\%) followed by Doxycycline (86\%), Gentamycin (82\%), Amikacin (79\%),
Vancomycine (68\%), Meropenem (61\%) and imipenem (54\%) and was resistant to Ciprofloxacin and Oflaxacin (64\%) and Cephradine $(50 \%)$ respectively as presented (Figure 1).

\section{Discussion}

The purpose of the current research was to evaluate the antibiogram of $S$. aureus from clinical specimens at Ayub Medical Complex. Abb0ttabad Pakistan. Maximum $S$. aureus numbers (40\%) were from pus specimen. This is similar with a former study done at KNH [14]. A research conducted to determine the antimicrobial profile of $S$. aureus strains isolated from patients in Iran, most of the isolates were from blood specimens (29\%) [15]. other investigation carried out on prevalence and antibiogram of $S$. aureus from clinical isolates in Nigeria revealed a mainstream of the isolates were from urine (76\%) [16]. Prominent number of $S$. aureus isolates from pus may be due to exposure of wounds which makes them more predisposed to infections and poor hygiene. S.aureus isolates were sensitive to Vancomycin and amikacine this findings are also in agreement with the results of Wankhade (17). In our study, MRSA was tested using Oxacillin screening. MRSA isolates were resistant to Imipenem (82\%), Ciprofloxacin (73\%), Oflaxacin (71\%) and Erythromycin (67\%). This is reliable with previous study completed at Ayub Medical Complex [18]. This may be due to the misuse of these antibiotics in daily practice at local hospitals. In our study $14 \%$ of MRSA isolates were resistant to Vancomycin. This result is somehow greater to a research in Iran which revealed $5 \%$ of the MRSA isolates were resistant to Vancomycin [19]. In the existing study MRSA was most common in blood $(45.45 \%)$ parallel finding was also detected earlier in study done in Lahore [20]. Although methicillin resistance and resistance to novel beta lactam antibiotics is due to mecA gene and its exhibition [21]. The 
study evaluated that furthermost potential drug against MRSA is Amikacin (95\%) followed by Vancomycin (86\%).In the current study on this basis of Oxacillin $(0 \%)$ sensitivity MRSA and MSSA were distinguished which is almost same as the study completed in KNH [14] and Iran [22]. This study exhibited that MSSA isolates were resistant to Ciprofloxacin (64\%), Oflaxacin (64\%) and fruitful drug against MSSA is
Oxacillin (100\%) followed by Doxycycline (86\%), Gentamicin (82\%) and Amikacin $(79 \%)$.

The study demonstrated that the isolates of S.aureus were mostly sensitive to Amikacin (86\%) followed by Doxycyclin (78\%), Vancomycin (76\%) and Gentamicin (72\%). On the other hand the S.aureus showed significant amount of resistant against Oflaxacin, Ciprofloxacin and Imipenem.

Table 1. Detail frequency of $S$.aureus isolates among different clinical samples

\begin{tabular}{|c|c|c|c|c|c|c|c|c|}
\hline Specimen & Media & $\begin{array}{c}\text { Total } \\
\text { sample }\end{array}$ & $\begin{array}{c}\text { Total } \\
\text { growth }\end{array}$ & Growth \% & MRSA & $\begin{array}{c}\text { \% OF } \\
\text { MRSA }\end{array}$ & MSSA & $\begin{array}{c}\text { \% OF } \\
\text { MSSA }\end{array}$ \\
\hline Urine & CLED & 200 & 25 & $25 \%$ & 10 & $22.7 \%$ & 15 & $26.78 \%$ \\
\hline Blood & MacConkey & 200 & 35 & $35 \%$ & 20 & $45.45 \%$ & 15 & $26.78 \%$ \\
\hline Pus & Blood agar & 200 & 40 & $40 \%$ & 14 & $31.81 \%$ & 26 & $46.42 \%$ \\
\hline \multicolumn{2}{|c|}{ Total } & 600 & 100 & & 44 & & 56 & \\
\hline
\end{tabular}

MRSA: Methicillin-Resistant Staphylococcus aureus; MSSA: Methicillin-Sensitive Staphylococcus Aureus

Table 2. Antimicrobial susceptibility profile of S.aureus

\begin{tabular}{|c|c|c|c|c|}
\hline S. No. & Antibiotics & Sensitive & Resistant & \% Sensitive \\
\hline 1 & Levofloxacin & 58 & 42 & $58 \%$ \\
\hline 2 & Ciprofloxacin & 32 & 68 & $32 \%$ \\
\hline 3 & Oflaxacin & 24 & 76 & $24 \%$ \\
\hline 4 & Erythromycin & 46 & 54 & $46 \%$ \\
\hline 5 & Doxycycline & 78 & 22 & $78 \%$ \\
\hline 6 & Oxacillin & 56 & 44 & $56 \%$ \\
\hline 7 & Gentamicin & 72 & 28 & $72 \%$ \\
\hline 8 & Amoxicillin & 54 & 46 & $54 \%$ \\
\hline 9 & Amikacin & 86 & 14 & $86 \%$ \\
\hline 10 & Cephradine & 50 & 50 & $50 \%$ \\
\hline 11 & Imipenem & 38 & 62 & $38 \%$ \\
\hline 12 & Meropenem & 52 & 48 & $52 \%$ \\
\hline 13 & Vancomycin & 76 & 24 & $76 \%$ \\
\hline
\end{tabular}




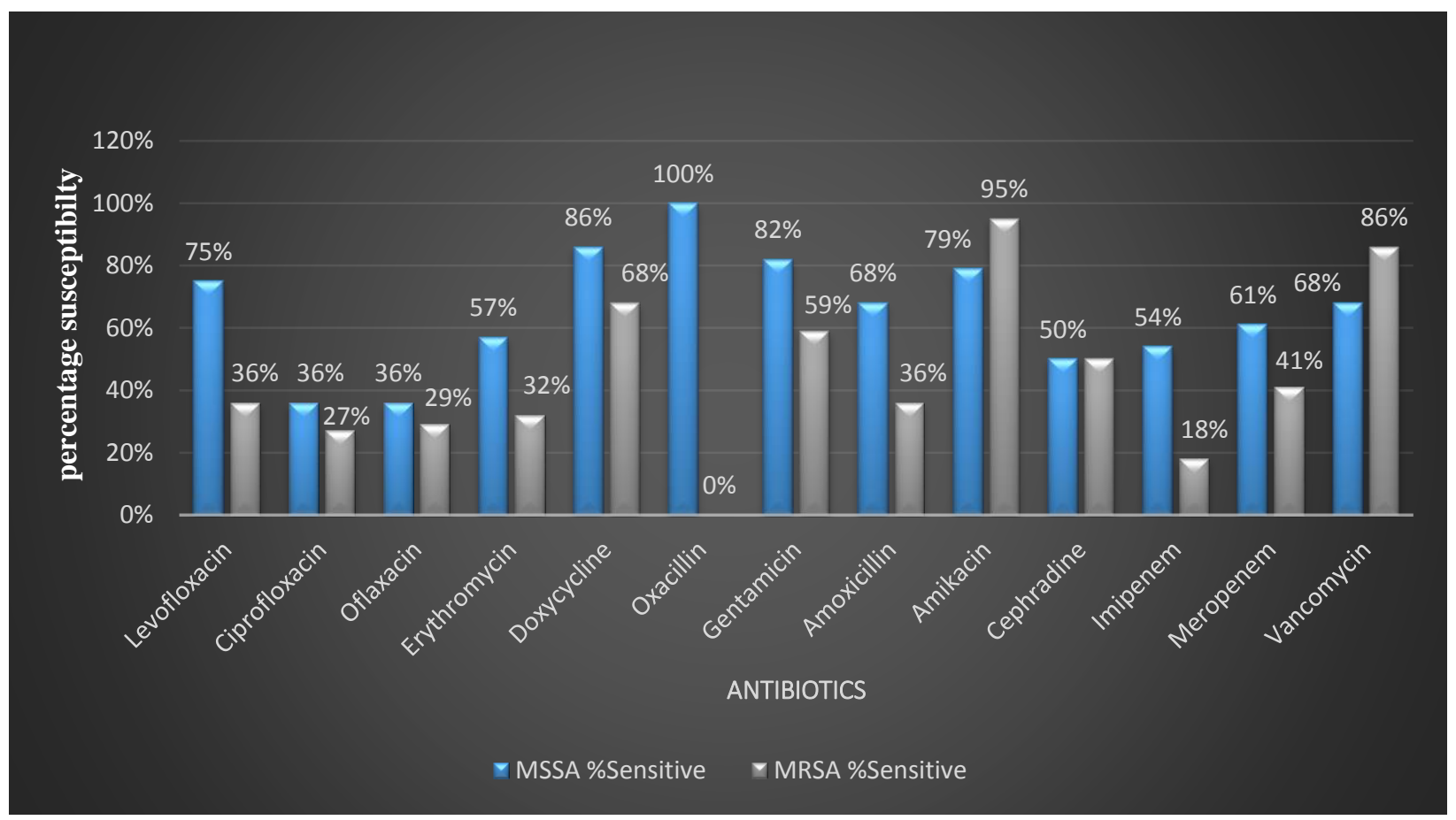

Figure 1. Comparison of Antimicrobial susceptibility profile of MRSA and MSSA

MRSA: Methicillin-Resistant Staphylococcus aureus; MSSA: Methicillin-Sensitive Staphylococcus Aureus

\section{Conclusion and recommendations}

Present study investigated that Staphylococcus aureus is one of the most vital pathogen isolated from most of the clinical specimen like pus, urine and blood. All the isolates were sensitive to Amikacin, Doxycyclin and Gentamicin. Hence endure the choice for S.aureus. It had also shown multidrug resistant pattern. So, systematic antimicrobial susceptibility surveillance is vital for area wise observing of the resistant pattern. This will be beneficial to realm the efficacy of antibiotics for healthier patient management. There is need for further exploration to evaluate the genes accountable for resistance and epidemiology of multiple drug resistant S.aureus and MRSA. Some information such as date of collection, patient age, gender, clinical information, prior antibiotics use and period of patient visit in the hospital were omitted.

\section{Authors' contributions}

Conceived and planned the project and supervised: MA Khan, Performed the experiment: S Jamil, Analyzed the data: S Ali \& IA Syed, Helped in proofreading of the manuscript: Z Ahmad.

\section{Acknowledgments}

The authors are thankful to the Lab supervisor of Ayub Medical College for providing financial aid to conduct this study. The authors also acknowledge the laboratory staff for their support and collaboration.

\section{References}

1. Pantosti A, Sanchini A \& Monaco M (2007). Mechanisms of antibiotic resistance in Staphylococcus aureus. J F Microbiol 2: 323-34

2. Loomba PS, Taneja J \& Mishra B (2010) Methicillin and vancomycin resistant $S$. aureus in hospitalized patients. J Glob Infect Dis 2: 275-83.

3. Donlan RM \& Costerton JW (2002). Biofilms survival mechanisms of clinically relevant microorganisms. Clin Microbiol Rev 15(2): 167-193

4. Funke G et al. (2011). Manual of Clinical Microbiology, 10th ed. ASM Press,

5. Espedido BA \& Gosbell IB (2012).Chromosomal mutations involved in antibiotic resistance in Staphylococcus aureus. Front Biosci 4: 900-15 
6. Enright MC, Robinson DA, Randle G, Feil EJ, Grundmann H \& Spratt BG ( 2002). The evolutionary history of methicillin-resistant Staphylococcus aureus (MRSA). Proc Natl Acad Sci 99(11): 7687-92.

7. Marlowe EM \& Bankowski MJ (2011). Conventional and molecular methods for the detection of methicillin-resistant Staphylococcus aureus. J Clin Microbiol 49(9 Suppl): S53-S6.

8. Anwar MS, Jaffery G, Bhatti KR, Tayib M \& Bokhari SR (2004). Staphylococcus aureus and MRSA nasal carriage in general population. J Med Microbiol 14: 661-664

9. Naqvi ZA, Hashmi K \& Kharal SA (2007). Methicillin resistant Staphylococcus aureus (MRSA) in burn patients. Pak J Pharmacol 24(2): 7-11

10. Khan S, Rasheed F \& Zahra R (2014). Genetic polymorphism of agr Locus and antibiotic resistance of Staphylococcus aureus at two hospitals in Pakistan. Pak $J$ Med Sci 30(1): 172

11. Costelloe C, Metcalfe C, Lovering A, Mant D \& Hay AD (2010). Effect of antibiotic prescribing in primary care on antimicrobial resistance in individual patients: systematic review and meta-analysis. BMJ 340: c2096

12. Blanc D, Lugeon C, Wenger A, Siegrist H \& Francioli P (1994) .Quantitative antibiogram typing using inhibition zone diameters compared with ribotyping for epidemiological typing of methicillinresistant Staphylococcus aureus. J Clin Microbiol 32(10): 2505-9.

13. Marlowe EM \& Bankowski MJ (2011).Conventional and molecular methods for the detection of methicillin-resistant Staphylococcus aureus. J Clin Microbiol 49(9 Suppl): S53-S6

14. Kanaga EL (2014). Antimicrobial susceptibility of bacteria that cause wound sepsis in the paediatric surgical patients at Kenyatta national hospital. http://erepo sitor y.uonbi .ac.ke/handl e/11295/95412.

15. Soltani R, Khalili H, Rasoolinejad M \& Abdollahi A (2010). Antimicrobial susceptibility pattern of Staphylococcus aureus strains isolated from hospitalized patients in Tehran, Iran. Iran J Pharm Sci 6: 125-32.

16. Obiazi HAK, Ekundayo AO \& Ukwandu NCD (2007). Prevalence and antibiotic susceptibility pattern of Staphylococcus aureus from clinical isolates grown at 37 and $44{ }^{\circ} \mathrm{C}$ from Irrua, Nigeria. African $J$ Microbiol Res 1: 57-60.

17. Wankhade $A B$, Panda,S, Hathiwala $R$ \& Keche Y (2017). Study of antibiotic resistance profiling of Staphylococcus aureus isolated from clinical specimens of the patients attending a tertiary teaching hospital from Chhattisgarh. Int J Res Med Sci 5(11): 4808-4811

18. Hassan AK, Mohammad M, Humera K, Samina N, Ahmed AK, Fridoon JA \& Riffat M (2014) Prevalence, antibiotic susceptibility pat-tern and demographic factors related to methicillin resistant Staphylococcus aureus in Lahore, Pakistan. Int J Microbiol Adv Immunol 2: 45-48.

19. Arianpoor A, Estaji F, Naderinasab M \& Askari E (2015). Antimicrobial susceptibility pattern of Staphylococcus aureus Isolates against newly marketed antibiotics. J Ayub Med Coll Abbottabad 3: 3-6.

20. Hassan AK, Mohammad M, Humera K, Samina N, Ahmed AK, Fridoon JA \& Riffat M (2014) Prevalence, antibiotic susceptibility pat-tern and demographic factors related to methicillin resistant Staphylococcus aureus in Lahore, Pakistan. Int J Microbiol Adv Immunol 2: 45-48.

21. Taj R, Muhammadzai I, Ahmad J, Khan A, Syed F \& Khan Z (2015). Frequency and antibiotic susceptibility pattern of methicillin resistant staphylococcus aureus in Abbottabad city of Pakistan. KMUJ (7): 4.

22. Arianpoor A, Estaji F, Naderinasab M \& Askari E (2015). Antimicrobial susceptibility pattern of Staphylococcus aureus Isolates against newly marketed antibiotics. J Ayub Med Coll Abbottabad 3: 3-6. 